\title{
1977 MEMBERSHIP DIRECTORY
}

(Corrected to February 1, 1977)

Permission for Commercial Use Must Be Obtained From

the NALLD Executive Secretary

A/V LANGUAGE JOURNAL, Birmingham B4 7ET, England

ABDUR-RAZZAQ, ABDULLAH H., Headmaster, Al-Karim School, 837 Park Place, Brooklyn, NY 11216

ABEL, JEAN P., Rd. 2 Martin Hill Road, Corning, NY 14830

ABEYTA, JERRY, Box 674, Center, CO 81125

AVADIA UNIVERSITY, Vaughn Memorial Library, Wolfville, NS Bop 1X0, Canada

ADAMS, MRS. SUDIE M., Baylor University, Spanish and Portuguese, Box 159 Waco, TX 76703

ADFL BULLETIN, Editor, 62 Fifth Avenue, New York, NY 10011

AIKENS, H. F., Director, Dalhousie University, Language Laboratory, Halifax, NS Canada

ALLEN, ERNEST, Director, Texas Christian University, Language Laboratory, Allen Farm Road, Ft. Worth, TX 76116

ALLEN, TED, Laboratory Director, ELS Language Center, UD Station, Irving, TX 75061

ALLIANCE FRANCAISE EAST BAY, Box 11138, Oakland, CA 94611

ALTMAN, DR. HOWARD B., University of Louisville, Dept. Modern Language, Louisville, KY 40208

ALTMAN, LEON, Director, San Francisco State College, Foreign Language Laboratory, 1600 Holloway, San francisco, CA 94132

AMER COUN TEACH FOR LANCS, Editor, For. Lang. Annals, 2 Park Ave., Room 1814, New York, NY 10016

ANASA, VILLETTE, Adelphi University; Language Laboratory, Garden City, NY 11530

ANDREWS UNIVERSITY, Chairman, Dept. Modern Language, Berrien Spgs., MI 49704

ANDREWS, M. L., Language Lab Supervisor, University of Otago, Box 56, Dunedin, New Zealand

APPALAChIAN STATE, Periodicals Department, University Library, Boone, NC 28608

APPLIED LIGUISTICS, INST. OF WILLY MARTIN, Blijde Inkomststraat 21, 3000 Leuven, Belgium

ARIZONA STATE UNIVERSITY, Library Periodicals, Tempe, AZ 85281

ASSELIN, JEAN-GUY, Supervisor, Carleton University, Language Laboratory, 126 Paterson Hall, Ottawa, ON KIS 5B6, Canada

ASSELT, IAN VAN, Head, McPherson College, Dept. Foreign Languages, McPherson, KS 67460

ASSOCIATION FOR EDUCATIONAL COMMUNICATION AND TECHNOLOCY, John J. Faber, Advertising Manager, 1201 Sixteenth St., N.W., Washington, DC 20036

ATTIC, CECIL, Supervisor, Language Lab, California State College, 800 N. State College Blvd., Fullerton, CA 92634 
AUCOIN, G. E., 34 Langley Ave., Dartmouth, NS B2W 2Y6, Canada

AULESTIA, VICTOR H., Director, University of Maryland, Language Media Center, 5401 Wilkens Avenue, Baltimore, MD 21228

AUSTRALIA, NAT'L LIBRARY OF PRELIMINARY PROCESSING, (NS 110/72), Canberra, A.C.T. 2600, Australia

AUSTRALIAN NAT'L UNIV., Instructional Resources Unit, Chifley Library, Box 4, Canberra A.C.T. 2600, Australia

AVEDEX INC., 7326 Niles Center Road, Skokie, IL 60076

BABIN, J. W., Assoc. Prof. French, St. Francis Xavier Univ., Antigonish, NS B2G 1 CO, Canada

BAKER, REID E., Ohio Dept. of Education, 65 S. Front Street, Columbus, OH 43215

BALL STATE UNIVERSITY, Continuations, Dept. of Library Service, Muncie, IN 47306

BALLEW, EMILY MAE, Instructor, Northwest Mississippi J. C., Modern \& Foreign Languages, Senatobia, MS 38668

BAO, BENJAMIN C. P., Language Lab Director, Agnes Scott College, B. Box 916, Decatur, GA 30030

BARR, CHARLES J., Director, St. John's College, Language Laboratory, 5118 Seminary Road, Camarillo, CA 93010

BAUMAN, MR. RONALD A., Dept. of Modern European Langs., Pomona College, Claremont, CA 91711

BECK, FRANCES J., University of Chicago, 5832 South Stoney Island, Chicago, IL 60637 BENT, ANTHONY J., 140 Brandeis Road, Newton, MA 02159

BERKELEY HIGH SCHOOL, C/O H Lorenzo, Foreign Languages, Grove \& Bancroft, Berkeley, CA 94704

BERLIN, FREE UNIVERSITY OF, Foreign Language Laboratory, Habelsch Werdter Allee 45 Ihnenstrasse 24, 1 Berlin 33 (Dahlem), Germany

BERUBE, JEANETTE, SISTER, Director, Annhurst College, Language Laboratory, Woodstock, CT 06281

BETTLER, MICHAEL, Director of Courses, ELS Language Center, 5009 Calhoun Road, Houston, TX 77004

BLAKE, LORRAINE J., Language Lab Director, Eastern Nazarene College, 23 East Elm St., Quincy, MA 02170

BOUDREAU, JACQUELINE, Supervisor, Wheaton College, Language Laboratory, Norton, MA 02766

BOUGARD, DR. ROGER, Lab Director, NCCU, Box 19371, Durham, NC 27705

BOWEN, LOUISE H., Dartmouth College, 201 Bartlett Hall, Hanover, NH 03755

BOWIE STATE COLLEGE, Thomas G. Pullen Library, Periodical Dept., Bowie, MD 20715

BOYDEN, PATRICK C., Director, Kent State University, Language Laboratory, Kent, $\mathrm{OH}$ 44240

BRANNOCK JR., D. V., Assistant Professor, Clemson University, 201 Strode Tower, Clemson, SC 29631 
BRAXTON JR., SHERROD L., Assistant Professor, French, Oral Roberts University, 7777 S. Lewis, Tulsa, OK 74136

BRESNICK, SOL, Coll. Lab. Technician, Queens College, 65-30 Kisseng Blvd., Flushing, NY 11367

BRIDGERS, MR. W. K., Comm. Prod. Asst., University of California, Phelps Hall 1521, Santa Barbara, CA 93106

BRIGCS, BECKY A., Michigan State University, Language Laboratories, A-126 Wells Hall, East Lansing, MI 48824

BRIGHAM YOUNG UNIVERSITY, Harold Lee Library, Serials Section, Provo, UT 84602

BRISTOL COMMUNITY COLLEGE, Library, 777 Elsbree Street, Fall River, MA 92720

BRITISH LIBRARY, Accessions Department, Lending Division, Boston Spa, Wetherby, Yorkshire LS23 7BQ, England

BRONX COMMUNITY COLLEGE, Library and Learn. Res. Ctr., Mrs. Annette Perctz, Media Librarian, W. 181 st St. and Univ. Ave., Bronx, NY 10453

BROSSMAN, DR. LUTHER F., Director, Albright College, Modern Language Laboratory, Reading, PA 19604

BROWN UNIVERSITY, Language Laboratory Library, Box E, Providence, RI 02912

BROWN, C. P., Western Kentucky University, Ivan Wilson Building 251, Foreign Language Department, Bowling Green, KY 42101

BUCHHANDLUNG LEHMKUHL, 8000 Munchen 23, Leopoldstraase 45, West Germany

BURCGRAAF, SAMUEL E., Chairman, Dept. of Learning Res. Ctrs., Brigham Young University, 2330 Lee Library, Provo, UT 84602

BUTLER, MRS. HORTENSIA, Asst. Dean, Foothill College, Mountain View Center, 1965 San Ramon Ave., Mountain View, CA 94043

CALIF POLYTECHNIC STATE UNIV., University Library, Periodicals Section, Sn Luis Obspo, CA 93407

CALIF. STATE UNIV.-SACREMENTO, Spanish-Portuguese Dept., 6000 J. Street, UPO No. 257, Sacremento, CA 95819

CALIF-SAN DIECO, Univ. of Language Lab C-008, Muir College, La Jolla, CA 92093

CALLAHAN, DR., 820 North Michigan Avenue, Chicago, IL 60611

CALVIN LIBRARY, 3207 Burton S.E., Grand Rapids, MI 49506

CAPE BRETON, College of Sydney Campus, Library, Box 760, Sydney, NS B1P 611, Canada

CAPRETZ, PIERRE J., Director, Yale University, Language Laboratory, 111 Grove Street, New Haven, CT 06510

CARLETON COLLEGE, Library, Northfield, MN 55057

CARLSON, FLORENCE, Director, Wellesley College, Language Laboratory, Wellesley, MA 02181

CARSON-NEWMAN COLLEGE, Library, Jefferson City, TN 37760

CARTIER JR., F. A., 1029 forest Avenue, Pacific Grove, CA 93950

CATE, ROBERT L., Director, University of Texas-EL Paso, Modern Language Laboratory, El Paso, TX 79968 
CENTRAL STATES CONFERENCE, William Clapper, Roanoke County Schools, 526 College Avenue, Salem, VA 24153

CHANC, GERALD K. J., University of Hawaii, Foreign Language Laboratory, Moore Hall 225, 1890 East-West Road, Honolulu, HI 96822

CHAPMAN, DAVID CRAIC, Learning Resources, University of California, Santa Barbara, CA 93106

CHEATHEM, ROSALIE M., Director, Univ. of Arkansas-Little Rock, Language Laboratory, 6405 Evergreen Road, Little Rock, AR 72207

CHERRY, CHARLES MAURICE, Director, Furman University, Modern Language Laboratory, Greenville, SC 29613

CHRIST, FRANK L., Coordinator: Lang Assistant, Support System, California St. UnivLong Beach, Long Beach, CA 90720

CHRISTMAN, HENRY R., Director, Kutztown State College, Language Laboratory, Kutztown, PA 19609

CICERAN, A. J., Supervisor, Brock University-Decew Campus, Language Laboratory, St. Catharines, ON L2S-3AI, Canada

CLEVELAND STATE UNIVERSITY, Library Order Unit-Serials, 1860 E. 22nd Street, Cleveland, OH 44115

COE, GAYLE, Audio-Visual Technician, Cleveland State University, 3487 W. 122 St., Cleveland, $\mathrm{OH} 44111$

COLORADO, UNIVERSITY OF LIBRARY, Serials, Boulder, CO 80309

COMET, HONORE, Director, All-World Language Institute, Language Laboratory, 208 South Beverly Drive, Beverly Hills, CA 90212

CONCEPCION, UNIVERSIDAD DE, Biblioteca Central, Casilla 1807, Concepcion, Chile

CONCORDIA UNIVERSITY, Sir George Williams Campus, Library, Serials Acquisitions, 1455 Maisonnouve Blvd., West, Montreal, Quebec H3G 1M8, anada

CONNORS, PAUL R., Coordinator, Foreign Languages, 37 Pine Grove Avenue, Sharon, MA 02067

CONWELL, DR. MARILYN J., Director, Rosemont College, Language Laboratory, 909 Concord Building, 251 W. Dekalb Pike, King of Prusia, PA 19406

COOPER, C. T., Assistant Professor-Spanish, Lebanon Valley College, Annville, PA 17003

CORTES, ELADIO, Director, Rutgers University, Spanish Department, Chairman, Camden, NJ 08682

COUROUX, GERALD, College Marie-Victorin, 7000 Rue Marie Victorin, Montreal, PQ 462, Canada

COX, JERRY, Director of Language Lab, Furman University, Dept. of Modern Foreign Langs., Greenville, SC 29613

COX, SHIRLEY O., Director, Auburn University, Language Laboratory, Auburn, AL 36830

CREAMER JR., John F., Director, Language Lab, Hudson High School, Brigham St., Hudson, MA 01749

CRIMINALE, LEONARD R., Director, Elmira College, Language Laboratory, Elmira, NY 14901

CROOKS, LAUREL, 8 Storrs Road, Hanover, NH 03755 
CTR FOR INFO ON LANG TEACH, Deputy Librarian, 20 Carlton House, Terrace, London S.W.I., England

DAI, HIROSHIMA, Honbu Toshokan-K, Hiroshima $730 \mathrm{MZ}$, Japan

DAIGAKU, GIFU JYOSHI TANKI, Toorin Nagara, GIFU 500 MZ, Japan

DAIGAKU, KYOTO SANCYO, Toshokan (Eigo-Ken), Motoyama Kamigamo, Kita-Ku, Kyoto $603 \mathrm{MZ}$, Japan

DAIGAKU, TOKOYO-SEITOKU- TANKI, Library (Mz), 7-12, Jujodai-L-Chowe, Kita Ku, Tokyo, Japan

DARIEN HIGH SCHOOL, Foreign Language Dept., Darien, CT 06820

DAVILLA, ADRIAN, Director, ELS Language Center, Laboratory, 5009 Calhoun Road, Houston, TX 77004

DAVIS, JAMES C., Language Laboratory, Humanities Hall 263, University of California, Irving, CA 92717

DAY, STEPHEN, Assistant Professor-French, Queen's University, Kingston, ON, Canada

DAYTON, UNIVERSITY OF LIBRARY, Periodicals Department, Dayton, OH 45469

DE GERY, MRS. X., Crystal Springs School-Girls, 400 Uplands Drive, Hillsborough, CA 94010

DE LA SAlLe COLleGE, Library, P.O. Box 3819, 2401 Taft Avenue, Manila, Philippines

DEATIE, DR. B. A., Cleveland State University, Instructional Media Servs Lab., Univversity Tower 807 , Cleveland, $\mathrm{OH} 44115$

DEBONE, DR. G., Head, Lagrange College, Language Department, Lagrange, GA 30240

DELANOEYE, ROSA E., Language Lab Director, California St. University-LA, 2400 Lyric Avenue, Los Angeles, CA 90027

DeLATTRE, MME E., Crapel-Cretaal, Univ. De Nancy II, B.P. 33.97, 54015 Nancy Cedex, France

DELONG, EDWARD JAMES, University of Richmond, Language Laboratory, 6634 Three Clopt Road, Richmond, VA 23226

DETI, DANIEL V., Chairman Language Department, College of the Desert, Palm Desert, CA 92260

DETORRE, EMILIO E., Queens College, CUNY, Flushing, NY 11367

DEURLOO, MRS. W. C., University of Alberta, Romance Language Lab, 24 Arts Building, Edmonton, AB, Canada

DICKERMAN, WILLIAM C., 16439 Brookford, Houston, TX 77058

DIO, Procurement Division, Attn: Mrs. Connor/XL, PO Box 325, Ft. ORD, CA 93941

DIVINCENZO, VITA J., Director, Villanova University, Language Laboratory, Spring \& Hilldale Road, Malvern Rd. 2, PA 19355

DLIWC ACADEMY LIBRARY, DAKFO3-77-M-3705, Building 302, Pres/Mtry, CA 93943

DOCK, STEPHEN V., Coordinator, Vanderbuilt University, Vanderbuilt Language Lab, Box 1651, Station B, Nashville, TN 37235

DODD, KEN, West Georgia College, School of Arts \& Sciences, Dept. of Foreign Languages, Carollton, GA 30117 
DOWLING, DR. J. K., Director, University of Queensland, Language Laboratory, St. Lucia, Brisbane 4067, Australia

DROBNIC, KARL, Lab Instructor, Eli, Oregon St. University, PO Box 487, Waldport, OR 97394

DUKAS, VYTAS, San Diego State College, Language Laboratory, 5402 College Avenue, San Diego, CA 92182

DULKA, RICHARD, Eastern Illinois University, Foreign Languages, Charleston, IL 61920

DUNAWAY, AGNES, Chairman, Riverside High School, Foreign Language Department, 1615 East Locust Street, Milwaukee, WI 53211

DYKEMA, MR. C. R., Youngstown State University, Foreign Languages, 410 Wick Avenue, Youngstown, $\mathrm{OH} 44503$

EASTERN MONTANA COLLEGE, Library, Serials Department, Billings, MT 59101

EBB, MS. NINA Z., 157 A South Trail, Stratford, CT 06497

ECONOMOS, R. M., Pace University, 41 Park Row, New York, NY 10038

EDMONDS, ERIC A., 135 Fayette Street, Palmyra, NY 14552

EDUCATIONAL MEDIA DIVISION, Jim Goodin, P.O. Box 20604, 2812 Quail Plaza Drive, Oklahoma City, OK 73120

EK, JAMES D., Director, Western Michigan University, Language Laboratory, Kalamazoo, MI 49008

EL PASO PUBLIC SCHOOLS, West Area Office, C/O S. Sigueiros, 2231 Arizona Street, El Paso, TX 79930

EL.DRIDGE, MR. RICHARD H., Dept. of Humanities, Florida Memorial College, 15800 NW 42nd Ave., Miami, FL 33054

ELMENDORF, WILLIAM E., Director, Western Washington State Col., Language Laboratory, Bellingham, WA 98225

ERIC EXCHANGE, LR/, Center for Afplied Linguistics, 1611 N. Kent Street, Arlington, VA 22209

ESPOSITO, A. F., Atlantic Union College, S Lancaster, MA 01561

FAULKNER, JAMES C., State Univ. of NY Teachers C., 17 Cedar Ridge Road, New Paltz, NY 12561

FECTEAU, LEO J., Chairman, Barrington High School, Foreign Language Department, Barrington, RI 02806

FELSHER, DR. WILLIAM, Chairman, University of Evansville, Foreign Language Department, P.O. Box 329, Evansville, IN 47702

FICARRA, A. V., Assistant Professor, Western Conn. St. College, 181 White. Street, Danbury, CT, 06810

FINN, BARTHOLOMEW J., Director, Skill Center, Language Laboratory, 1485 Market Street, San Francisco, CA 94103

FISH, WILLIAM M., ELS Language Center, Wentworth College, Boston, MA 02115

FISHER, RONALD D., Director, LaSalle College, Lancaster, Laboratory, 20th Street at Olney Avenue, Philadelphia, PA 19141

FITCH, BRUCE C., Director, Transylvania College, Language Laboratory, 300 North Broadway, Lexington, $\mathrm{KY} 40508$ 
FITCHBURC HIGH SCHOOL, Attn: John C. Baimas, F. L. Curriculum Leader, 98 Academy Street, Fitchburg, MA 01420

FLORIDA STATE UNIVERSITY, Serials Department, R. M. Strozier Librar, Tallahassee, FL 32306

FORD, PROF. A. F., Language Lab, Northeastern University, Room 406 DG, Boston, MA 02115

FRADKIN, BERNARD, Evergreen Valley College, 3095 Yerba Buena Road, San Jose, CA 95121

FRECHETTE, ERNEST A., DR., Florida State University, Foreign Language Education, Tallahassee, FL 32306

FRIEDMAN, FRANK M., Director, C. S. Mott Community College, Language Laboratories, 1401 E. Court Street, Flint, MI 48503

FRITSCH, HANS J., P.O. Box 61, Whately, MA 01093

FRITZ, ROBERT KARL, 2848 101st Place, Apartment 4C, Highland, IN 46322

FROST, ARTHUR F., Director, Princeton University, Language Laboratory, 304 E. Pyne Building, Princeton, NJ 08540

FROST, RAMONAZ, Instructor, Cuesta College, 215 Longview Lane, Sn Luis Obspo, CA 93401

FUKUOKA UNIVERSITY, LL Room, Nanakuma, Fukuoka 814 (MZ), Japan

GARFINKEL, ALAN, DR., Associate Professor, Purdue University, Dept. of Modern Lang. Educ., Lafayette, IN 47907

GARINGER, ERMAL E., Director, University of Kansas, Language Laboratories, Wescoe Hall, Lawrence, KS 66045

GAUDINO, V. A., Modern Language Center, 252 Bloor Street West, Toronto, ON 818, Canada

GEMMATO, GENO R., Director, 7 ufts University, Audio Labs, Eaton Hall L22, Medford, MA 02155

GeNDREAU, N., Rhode Island College, Modern Language Department, 600 Mount Pleasant Avenue, Providence, RI 02908

GENESEE COMMUNITY COLLEGE, Alfred C. O'Connell Library, Box 718 College Road, Batavia, NY 14020

GeNNAULA, M. G., 5872 Kings School Road, Bethel Park, PA 15102

GEORGE WASHINGTON UNIVERSITY, Romance Languages \& Lit., Washington, DC 20006

GEORGIA, UNIVERSITY OF LIBRARIES, Sets Department, Athens, GA 30602

GERMAN CENTER, BOSTON, Director, Language Division, 170 Beacon Street, Boston, MA 02116

GERMAN QUARTERLY, Editor, William A. Little, University of Virginia, Germanic and Slavic Lang., Charlottesville, VA 22901

GIELLA, MICUEL, ANGEL, Carleton University, Spanish Department, Colonel by Drive, Ottawa 1, ON, Canada

GILL, S. ROSENDA, Coordinator, Regis College, Language Laboratory, Weston, MA 02193 
GIONET, ARTHUR J., DR., North Texas State University, Dept. For. Langs. \& Lit., Denton, TX 76203

GLEAVES, MR. GLEN, Language Laboratory, University of S. Alabama, Mobile, AL 36688

GOLDSWORTHY, T. R., Director, Univ. of Wisconsin at Madison, Labs for Recorded Instruction, Van Hise Hall 279, 1220 Linden Drive, Madison, WI 53706

GOODWIN, ANNE M., MS., Lab Asst., Boston State College, 625 Huntington Ave., Boston, MA 02115

GORDON, DAVID L., Director, Marquette University, Dept. of Foreign Lang. \& Lit., Language Laboratories, 526 North Fourteenth Street, Milwaukee, WI 53233

GORE, PETER B., Director, Deerfield Academy, Language Laboratory, Deerfield, MA 01342

GOULET, NOELLA, SR., French Teacher, Notre Dame College, 2321 Elm Street, Manchester, NH 03104

GREAT FALLS, COLLEGE OF, LIBRARY, Periodicals Dept., 1301 20th Street South, Great Falls, MT 59405

GREEN, RUTH L., Director, William College, Language Laboratory, Karl E. Weston Lang. Center, Williamstown, MA 01267

GREGORY, CLARA, 2021 Bristlecone PI, The Woodlands, TX 77380

GRIGG, BILLIE, MRS., Supervisor, EI Camino College Lang. Lab., Torrence, CA 90506

GROSJEAN, GLEN, Assistant Director, University of California, Language Laboratory, B-40 Dwinelle Hall, Berkeley, CA 94720

GROSS, STEPHANIA E., Bowling Green State Univ., University Hall, Bowling Green, OH 43402

GUITTON, JEAN, DR., Chairman, Georgia College, Modern Language Laboratory, Milledgeville, GA 31061

CWIN, MARY E., Director, University of Mississippi, Language Laboratory, University, MS 38677

HALL, BUNELLE M., A-V Coordinator, Monterey Institute For Foreign Studies, 440 Van Buren Street, Monterey, CA 93940

HAMMERLY, HECTOR, Simon Fraser University, Applied Linguistics, Department of Modern Langs., Burnaby, BC V5A IS6, Canada

HAMMOND, VERNON, DR., Span. Lang. Publication Serv., 211 S. Main Street, McAllen, TX 78501

HANSON, ERWIN M., Director \& Lecturer, University of Michigan, Language Laboratory, 2003 Modern Lang. Building, Ann Arbor, MI 48104

HANDELSMAN, MICHAel, Asst. Prof., University of Tennessee, Dept. of Romance Languages, 601 McClung Tower, Knoxville, TN 37916

HANSON, DAVID A., Assistant Professor, Brandeis University, Listening Center, Waltham, MA 02154

HARJAN, PAULA, Lang. Lab. Supervisor, York University, Ross Bldg. 5115, 4700 Keile Street, Downsview, ON M3] 1P3, Canada

HARRISON, JOHN S., Foreign Languages, Board of Education of Baltimore County, Towson, MD 21222

HARTLEY, C. CHRISTOPHER, Head, The Peddie School, Language Department, Hightstown, NJ 08520 
HAWKINS, CORNELIA, Librarian, Ross Memorial Library, Cottey College, Ross Memorial Library, Nevada, MO 64772

HEALY, ANNE, Supervisor Language Lab, Boston College, Lyons 313, Chestnut Hill, MA 02167

HEATON, J., A-V Specialist-Lecturer, TesI Department, UCLA, Los Angeles, CA 90024

HEBREW UNIVERSITY, Center for Applied Lings, Popik Building, Jerusalem, Israel

HENDERICKSON, ROBERT L., 952 Nooney Street, Poplar Bluff, MO 63901

HENNEBERGER, ROBERT, Director, New College, University of Georgia, Language Laboratory, Athens, GA 30601

HENNIC, C. W., University of Waterloo, Language Laboratory, Room 110 ML Building, Waterloo, ON, Canada

HENRY, MARTIN A., MR., Director, Language Lab., Box 172, Gardiner, NY 12525

HERBERT, MR., Boise State University, 206 La Foreign Language, 1910 College Blvd., Boise, ID 83725

HERNANDEZ, ADELE B., Instructor, University of Maine, Department of Spanish, 96 Falmouth Street, Portland, ME 04103

HERNANDEZ, IRIS, Professor Pensacola Junior College, Language Laboratory, Pensacola, FL 32503

HERRERA, LAZARO M., Director, The Westminister Schools, Language Laboratory, 1424 W. Paces Ferry Road N.W., Atlanta, GA 30327

HEWSON, J., DR., Head, Memorial U. of Newfoundland, Department of Linguistics, St. John's, NF, Canada

HILTS, MRS. MARGARETE, Loma Linda University, La Sierra College, Riverside, CA 92505

HIRSCH, B. C., Director, Samford University, Language Laboratory, 800 Lakeshore Drive, Birmingham, AL 35209

HISPANA, Advertising Manager, Albert Turner, Glenbrook South High School, 4000 West Lake Avenue, Clenview, IL 60025

HISPANIA, Editor, Donald W. Blenznick, University of Cincinnati, Romance Languages, Cincinnati, $\mathrm{OH} 45221$

HOLMES, GLYN, DR., Director of Lang. Labs/French, Department of French, Univ. of Western Ontario, London, ON N6A3K7, Canada

HOWARD UNIVERSITY, Language Lab/903301, Room 361 Locke Hall, 2441 6th Street NW, Washington, DC 20059

HOWELL, JOEL T., Director, North Texas State University, Language Laboratories, Department of Foreign Langs., Denton, TX 76201

HOWLING, ROBERT T., Professor, New Haven College, English Department, New Haven, CT 06505

HUGHETT JR., HARVEY L., Instructor In Spanish, Virginia Polytech Inst \& S.U., Department of Foreign Langs., Blacksburg, VA 24060

HUTTEN, JEAN R., R.N., Coordinator of Learning Lab, Indiana University, School of Nursing, 1100 W. Michigan, Indianapolis, IN 46202

IANNUZZI, D.A., Chairman, Niagara University, Modern Languages, Niagara, NY 14109 INST FUR DOLMETSCHER AUSBILDUNG, Universitat Innsbruck, Innsbruck 6020, Austria 
INSTITUT DE LINGUISTIQUE, Appliquee, Laboratories De Langues, University De Strasbourg, 22 Rue Descartes, 67084 Strasbourg-Cedex, France

INSTRUCTIONAL MATERIALS CTR, 201 East Nine Mile Road, Highland Spgs, VA 23075

IRVING, EVELYN, DR., 1840 Azrock Drive, Knoxville, TN 37914

IZUMI, MASUKO, Director Language Lab, Seinan Gakuin University, 6-2-92 Nishijin Nishi-Ku, Fukuoka 814, Japan

JACKSON, GORDON, Director, Capital University, Language Laboratory, 2199 E. Main Street, Columbus, OH 43209

JARLETT, FRANCIS G., Language Laboratory Director, Central Conneticut State Col., Modern Language Department, New Britain, CT 06050

JENKINS, JACINTO, Language Laboratory Director, Calif. State Univ.-Sacremento, Dept. of Spanish \& Portuguese, 6000 J. Street, UPO 948, Sacremento, CA 95819

JOCUMS, GEORGE, DR., Chairman, Foreign Language Dept., Boise State University, 1910 University Drive, Boise, ID 83725

JOHNS, ALAN, Electronic Tech, Windsor University, French Lang \& Lit Department, Sunset Avenue, Windsor II, ON, Canada

JOHNSON, BARBARA, Washington State University, Foreign Language Department, Pullman, WA 99163

JORDAN, T. C., Director, Griffith University, Language Centre, Nathan Queensland 4111, Australia

KAGAWA UNIVERSITY, Saiwai-Cho. Takamatsu-Shi, 760 (Kin), Japan

KATH, RUTH ROBERT, University of lowa, lowa City, IA 52240

KATHOLIEKE UNIV. LEUVEN, Facult of Letters \& Phil., Dept. of Linguistrics, Section of Applied Ling., Blijde Inkomststraat 21, 3000 Leuven Belgium, Belgium

KAUPPAKORKEAKDULU, Runeberginkatu 14-16, Helsinki 10, Finland

KENNEDY, DORA F., Sufervisor, Prirce Georges's Public School, Foreign Language Department, 4806 Harvard Road, College Park, MD 20740

KENTUCKY, UNIVERSITY OF LANGUAGE LABORATORY, 312 Classroom Building, Lexington, KY 40506

KING, GAIL S., Director, Geddes Language Center, Boston University, 685 Commonwealth-Room 540, Boston, MA 02215

KIRJASTO, JCENSUUN KORKEAK, PL 111, 80101 Joensuu 10, Finland

KITAO, KENJI, Japan International Center, 39-1 Koyana- Shimofusa-Cho, Kita-uu, Koyto, Japan

KOENEN, SHIRLEY, 2420 Appleside Blvd., Clarkston, WA 99403

KCMPLEKTOVANIA, BURO, Minist Vyssh OBRAZ, Lengori B-234, Moscow, USSR

KONRAD, KARL-LUDWIG J., Director, Eastern Illinois University, Language Laboratory, Charleston, IL 61920

KONWTTAR, EGYETEMI, Pob 483, Budapest 1372, Hungary

KOPPEL, HELEN A., Director, Audio Laboratory/M73716, Tufts University, Eaton HallRoom 122, Medford, MA 02155

KORKEAKOULU, JOENSUUN, Kirjasto, Tulliportinkatu, 1A, 80130 Joensuu 13, Finland 
KOVARY, TOM T., Director, International Communications, Suny-Cortland, Cortland, NY 13045

KOZORIZ, G., Commandant, Canadian Forces FLS, Dept. of National Defense, Ottowa, ON 4, Canada

KRUSE, JR., E. L., 733 Kirkwood Drive, Grand Island, NY 14072

KRYNITZ, ROY L., Laboratory Director, New Mexico State University, Foreign Language Department, University Pk, NM 88070

KUMP, RICHARD J., 1822 Ricardo Drive, Cpe Girardeau, MO 63701

KUNKLE, JOHN F., DR., Director, $U$ of Southwestern Louisıana, Language Laboratory, 210 Elmwood, Lafayette, LA 70501

KURITA, KATSUMI, Sony Language Laboratory, 6-5-8 Kitashinagawa, Shinagawa-Ku, Tokoyo 141, Japan

KURIYAMA, SHOICHI, Language Laboratory, Waseda University, 1-6-1 Nishi-Waseda, Tokyo 160, Japan

KUTATOKOZPONT, FELSOOKTATASI, 1431, Budapest VIII Rigo U. 16, Peoples Republic of Hungary, Hungary

LACEFIELD, ARCH S., Senior Instructor, University of Kentucky, Henderson Community College, College Library/DAV 798100, Henderson, KY 42420

LALLY JR., DALE V., Director, University of Louisville, Arts \& Sciences Learning Lab, Hum-Room 16, Louisville, KY 40204

LAMiERAND, R., DR., 252 Bloor Street West, Toronto, ON M5SLV6, Canada

LANG \& LANG BEHAVIOR ABSTRACTS, P.O. Box 22206, San Diego, CA 92122

LANGE, DALE L., Professor, University of Minnesota, 148A Peik Hall, Minneapolis, MN 55455

LATHROP, THOMAS A., Transylvania College, 300 North Broadway, Lexington, KY 40508

LAWARSON, ROBIN E., DR., Director, Temple University, Media Learning Center, Philadelphia, PA 19122

LEMYZE, J. C., MCGill University, Language Laboratory, Peterson Hall Room 2, 3460 Mctavish, Montreal, PQ H3A 1XG, Canada

LEONE, ARTHUR A., 130 Roxbury Drive, Holiday, FL 33589

LETHBRIDGE, UNIVERSITY OF, LIBRARY, Serials Section, 4401 University Drive, Lethbridge, Alta T1K 3M4, Canada

LEVINSON, MRS. BERNICE, Director Language Laboratory, Brooklyn College, Cuny, Dept. of Modern Langs \& Lits, Brooklyn, NY 11210

LIBRARY OF CONGRESS, Ord. Division, Attn: Section C, Washington, DC 20540

LIDJI, MARCEL, Lecturer, City College of New York, Romance Language Laboratory, 133rd \& Convent Avenue, New York, NY 10031

LIFE, THOMAS E., Chairman, Union University, Language Department, Jackson, TN 38301

LIGHTCAP, DAVID W., Audio Visual Director, Susquehanna University, Selinsgrove, PA 17870

LINGUAPHONE INSTITUTE LTD., 207/209 Regent Street, London, England 
LOEW, RICHARD A.., Director, Language \& Learning Lab, 126 Clements Hall, Amherst, NY 14260

LONG BEACH CITY COLLECE, Foreign Language Department, 4901 East Carson Street, Long Beach, CA 90808

LOOK, R. KENT, Classroom \& Tech Staff Supr., U. of California-Berkeley, Language Laboratory, B-40 Dwinelle Hall, Berkeley, CA 94720

LORE, A. G., DR., Director, University of North Carolina, Language Laboratory, 105 Dry Hall, Chapel Hill, NC 27614

LOUISVILLE, UNIVERSITY OF BELKNAP CAMPUS, Attn: Mrs. Spradlin, Library, Serials Department, Louisville, KY 40208

LOYD, JEANNE, Instructional Assistant, Community College of Denver, North Campus, 1001 E. 62nd Avenue, Denver, CO 80216

I.UCKAU, PAUL F., Brigham Young University, Germanic \& Slavic Langs., 329 Mckb, Provo, UT $\mathbf{8 4 6 0 2}$

LUCKER, M.S., Language Lab, Bldg. No. 14N-0641, Mass. Inst. of Technology, Cambridge, MA 02139

MA, RICHARD W. C., Learning Asst. Cntr. Dir., Hawaii Pacific College, 841 Bishop Street, Honolulu, HI 96813

MACINKO, JOHN, Director, University of Colorado, Language Labs, 302 Old Main Building, Boulder, CO 80302

MACKEY, CHARLES R., Simmons College/Foreign Langs., 300 The Fenway, Boston, MA 02115

MACKEY, LOLA A., 1020 Crown Drive, Reno, NV 89503

MACILL, ROBERT, Director, Language Lab, Towson State University, Dept. of Modern Language, Baltimore, MD 21204

MAGIN, J. F., University of Western Ontario, London, ON 72, Canada

MANN SR., GARY D., Director, Ohio State University, Listening Center, Dieter Cunz Hall 155, Columbus, $\mathrm{OH} 43210$

MARESCHAL, ROGER E., 257 Avenue De La Colline, Aylmer, PQ J9J 1M2, Canada

MARIETTA COLLEGE, Modern Language Department, Marietta, OH 45760

MARINO, ANNA, Director, Hunter College, Language Laboratory, 695 Park Avenue Box 375, New York, NY 10021

MARQUETTE UNIVERSITY, Dept. of Foreign Languages, 526 North 14th Street, Milwaukee, WI 53223

MARTIN, WILLARD, Director, Listening-Learning Service, The Pennsylvania State Univ., 13 Pinchot Mall, University Park, PA 16802

MARXHEIMER, EDWARD, Assoc. Professor, University of Alberta, Dept. of Romance Langs., Edmonton, $A B$ T6C 2E1, Canada

MARYLAND, UNIVERSITY OF BALTIMORE COLLEGE, Library-Serials, 5401 Wilkens Avenue, Baltimore, MD 21228

MCANDREW, ALEX, Director, University of Sydney, Level 2 Brennan Building, Language Laboratory, Sydney 2006, New Australia

MCCLURG, AVERY, Dept. of Lit. \& Lang., East Texas State University, Commerce, TX 75428 
MCCULLOUGH, J. T., Professor, California State University, Spanish \& Portuguese Dept., 6000 J Street, Sacramento, CA 95819

MCCUNE, JERRY, Director, Indiana University, Language Laboratory, Ballantine Hall 120, Bloomington, IN 47401

MCGILL UNIVERSITY LIBRARIES, Serials Department, 3459 Mctavish Street, Montreal, PQ 112, Canada

MEDGAR EVERS COLLEGE, City University of New York, 1127 Carroll Street, Brooklyn, NY 11225

MEIJI GAKUIN UNIVERSITY, Gaigo-Ken, 2-37, Shiroganedai L-Chome, Minatoku, Toyko 108 (550), Japan

MELBORNE, UNIVERSITY OF, Language Centre Arts S, Parkville 3052, Victoria, Australia

MEMPHIS STATE UNIVERSITY, Department of Foreign Lang., Memphis, TN 38152

MENIN, J. L., Acting Director, Rice University, Language Laboratory, Rayzor Hall 106, Houston, TX 77005

MESSNER JR., C. A., Carleton College, Assoc. Prof. Mod. Lang., Northfield, MN 55057 METCALFE, JOHN, 29 Willow Rd., Menlo Park, CA 94025

MICKO, MICHALE, Director, Language Lab., Widener College, Chester, PA 19013

MILler, GLADYS, Occidental College, Supervisor, Language Lab, 1600 Campus Road, Los Angeles, CA 90041

MILleR, J. B., Assistant Professor, Wittenberg University, Springfield, OH 45501

MILLER, MARTHA R., Admin. Asst., Harvard University, Boylston Hall G-3, Cambridge, MA 02138

MILLER, ROBERT C., Chairman, Modern Language Department, The Thacher School, Ojai, CA 93023

MILLER, SHELBY C., 411 Putnam Ave., Cambridge, MA 02139

MILLER, WILLIAM I., DR., Asst. Prof. Modern Lang., University of Akron, 310 Olin Hall, Akron, $\mathrm{OH} 44325$

MINISTRY OF CULTURE \& RECREATION-RESOURCE CENTRE 77 Bloor St. West, 9th Floor, Toronto, ON M7A2R9, Canada

MINNESOTA, UNIVERSITY OF, Serial Records, Minneapolis, MN 55455

MINNESOTA, UNIVERSITY OF, Language Department, Duluth, MN 58812

MODERN LANG. JOURNAL, Editor, Robert F. Roeming, University of Wisconsin, Milwaukee, WI 53201

MODERN LANG. JOURNAL, Business Manager, Wallace G. Klein, 13149 Cannes Drive, St. Louis, MO 63141

MODERN LANGUAGE ASSOCIATION, Advertising Manager, 62 Fifth Ave., New York, NY 10011

MOLDIN, COLUMBUS M., Central Area Admin. Center, 6501 Lowland Drive, Landover, MD 20786

MONASH UNIVERSITY, Periodicals Department, Clayton VIX 3168, Australia

MOORE, J. MICHAEL, DR., Mesa College, San Diego, CA 92111 
MOOREHEAD STATE UNIVERSITY, Library-Serials Dept., Moorehead, MN 56560

MORENO, RAMON G. HERNANDEZ, Macuspana Public Schools, Dept. of English, Plaza De La Constitucion 107, Macuspana, Tabasco, Mexico

MORRISON, ROBERT R., Southern Missionary College, P.O. Box 475, Collegedale, TN 37315

MOSER, WORTH S., Darlington School, Rome, GA 30161

MOUNTAIN, CLIFF, Director, Foreign Languages, Mesa College, Grnd Junction, CO 81501

MULHOLLAND, CHARLES V., Supervisor, Lang. Lab., University of Rhode Island, Department of Languages, Kingston, RI 02881

MULLA, MR. MOHAMED, 2385-6 Bishop, Ann Arbor, MI 48105

MURPHY, HAROLD T., Asst. Prof., Marshall University, Box 238, 16th and Third Street, Huntington, WV 25701

NANYANG UNIVERSITY, Library/Period Sect., Upper Jurong Road, Singapore, 22, Republic of Singapore

NARVAEZ, LEON, Assistant Professor, Romance Languages, Saint Olaf College, Northfield, MN 55057

NASH, DONALD S., Roberts Wesleyan College, North Chili, NY 14514

NASSAU COMMUNITY COLLEGE, Library, Periodicals Unit, Attn: Ms. Magda Cantil, Stewart Avenue, Garden City, NY 11530

NATIONAL LIBRARY OF CANADA, Order Section Per 221424, 395 Wellington St., Ottawa, Ontario K1A ON4, Canada

NAVES, CARROLL E., Language Lab Director, Barry College, Miami, FL 33161

NEBRASKA, UNIVERSITY OF PERIODICAL DEPARTMENT, Gene Eppley Library, Box 688 Downtown Station, Omaha, NE 68101

NEUFCHATEL, UNIVERSITE DE, Chaire De Linguistique Gen., Neufchatel, 2000, Suisse NEVADA, UNIVERSITY OF, Library-Periodicals Dept., Las Vegas, NV 89109

NEVADA, UNIVERSITY OF, Library, Order Department, Reno, NV 89507

NEW MEXICO SCHOOL PROD. CO., P.O. Box 2126, Albuquerque, NM 87103

NEW YORK STATE UNIVERSITY, Language Lab Director, 3435 Main Street, 7 Hayes C, Buffalo, NY 14214

NEW YORK STATE UNIVERSITY, E. H. Butler Library, 1300 Elmwood Avenue, Buffalo, NY 14222

NEW YORK, STATE UNIVERSITY OF, LIBRARY, Serial Section, Vestal Parkway East, Binghamton, NY 13901

NEWCASTLE, University of Serials Librarian, New South Wales, 2308, Australia

NIEMAN, LINDA L., 11 Gary Place, Wappingrs Fls, NY 12590

NOBLE, DAVID, DR., Asst. Prof. German Dept., Armstrong State College, Savannah, GA 31406

NOJD, PROF. OLAVI, Director, Jyvaskyla University, Language Laboratory, SF-40 100 Jyuaskyla 10, Finland 
NORRISH, N., Director Lang. Lab., Victoria University of Wellington, Private Bag, New Zealand

NORTH HENNEPIN, Community College, Humanities Department, 7411 85th Avenue N, Minneapolis, MN 55445

NORTHEAST CONFERENCE, James W. Dodge, Secrettary Treasure, Box 623, Middlebury, VT 05753

NORTHROP WORLDWIDE AIRCRAFT, Services Inc., Attn: Librarian, Building 6237, Lackland AFB, TX 78236

NUFFER, J. STANLEY, Learning Lab Supervisor, Portland State University, P.O. Box 751, Portland, OR 97207

O'BRIEN, P. J., 280 Richmond Ave., Amityville, NY 11701

ODENSE UNIVERSITY, Language Laboratory, TSS Niels Bohr, Alle 75, DK-5000 Odense, Denmark

ODOM, WILLIAM, Department of Foreign Lang., Univ. of Southern Mississippi, Southern Station, Box 198, Hattiesburg, MS 39401

OHIO STATE UNIVERSITY, Serials Division, 1858 Neil Avenue, Columbus, OH 43210

OKLAHOMA BAPTIST UNIVERSITY, Director Language Lab., Shawnee, OK 74801

OKLAHOMA STATE UNIVERSITY, Library-Serials Division, Stillwater, OK 74074

ONTARIO INSTITUTE FOR STUDIES IN EDUCATION, Modern Language Center, Library-Periodicals, 252 Bloor Street W., Toronto, ON M5S 1V6, Canada

OUELLETTE, R. J., Director, Language Lab, Southeastern Massachusetts University, Old Westport Road, Group I Bldg., RM 207, N. Dartmouth, MA 02747

P/H ELECTRONICS, 117 East Helena Street, Dayton, OH 45404

P/H ELECTRONICS, 117 East Helena Street, Dayton, OH 45404

PARK DLL, WILLIAM M., DR., Foreign Language Dept., University of North Carolina, Charlotte, NC 28213

PATRON, GLORIA A., Univ. of New Orleans, Language Laboratory, Lake Front, New Orleans, LA 70122

PAUCHET, IACQUELINE, Director Language Lab., Knoxville College, Knoxville, TN 37914

PAUL, MRS. C. E., Philadelphia College of Pharmacy and Science, 43rd St. \& Kingsessing Ave., Philadelphia, PA 19104

PENNSYLVANIA STATE LIBRARY, Serials Records Section, Box 1601, Room 46, Harrisburg, PA 17126

PENSACOLA JR. COLLEGE, Library, 1000 College Blvd., Pensacola, FL 32504

PENTA, RICHARD M., Coordinator of Foreign Lang., Belmont School System, 38 Normandy Road, Lexington, MA 02173

PEREZ, MARICO P., Director Language Lab., Brevard College, Brevard, NC 28712

PESOLA, C. A., Media Director, Saint Olaf College, Northfield, MN 55057

PETERSEN, GERALD W., DR., Dept. of Foreign Languages, University of Nevada, Reno, NV 89507

PETHERBRIDGE, D. L., Assoc. Professor, University of Lethbridge, Lethbridge, AB, Canada 
PFLANZ, BARBARA, Associate Professor, University of Redlands, Languages \& Literatures, Redlands, CA 92373

PHILIPPON, CHRISTIAN P., 2707 Stuart Street, Berkeley, CA 94705

PIOLA, GLADYS C., Director of Language Lab., 106 Oakland Avenue Apt. A6, West Hartford, CT 06119

PLEASANTS, PROF. J. V., Columbia University, 20 Dewey Lane, Hampton Bays, NY 11946

PORT ELIZABETH, University of Library-Periodicals Dept., Private Bag 6058, Port Elizabeth 6000 , South Africa

PUERTO RICO, INTER-AMERICAN University, Library, San German, PR 00753

PURDUE UNIVERSITY, Serials Unit-Library, W. Lafayette, IN 37907

QUEENS UNIVERSITY, Paul Klapper Librar, Serials Section, Flushing, NY 11367

QUEENSLAND, University of Serials Librarian, Main Library, St. Lucia, Queensland 4067, Australia

QUICK, LEROY A., Northrop 307, APO, NY 09616

QUINN, T. J., Language Centre, Univ. of Melbourne, Parkville, Vic. 3052, Australia

RAMSAY, GEORGE, President, Instruction Systems, Inc., 985-D First Avenue, Des Plaines, IL 60016

RANDOLPH HIGH SCHOOL, Foreign Language Coordinator, Memorial Parkway, Randolph, MA 02368

REESE, L. GRANT, Language \& Philosophy, UMC No. 07, P.O. No. 35732, Utah State University, Logan, UT 84322

REESE, WINSTON J., Language Lab Director, University of lowa, 125 Schaeffer Hall, lowa City, IA 52240

REEVES, GAYNOR E., Director, Univ. of Newcastle 2308, Language Laboratory, Newcastle, New South Wales, Australia

REEVES, PROF. DONA, Modern Language Dept., Southwest Texas State Univ., Rt. 1, Box 239A, Buda, TX 78610

RHODESIA, University of Language Laboratory No. 3643, P.O. Box MP 167, Mount Pleasant, Salisbury, Rhodesia

RICE, THOMAS, 11952 Bornite Avenue, Hesperia, CA 92345

RICHMOND, EDMUN B., DR., Assistant Professor of German, Georgia Inst. of Technology, Atlanta, GA 30332

RICHTER, JOCHEN, Director of Language Lab, Allegheny College, Meadville, PA 16335

RICHTERICH, RENE, Director, Abteilung Fur Angewandte, Linguistik, Hallerstrasse 12, 3012 Berne, Switzerland

RIDDLE, BENTON E., Language Lab Director, Highland Park College, Highland Park, MI 48203

RIJKUNIVERSITEIT TE, Groningen, Bibliotheek, Oude Kijk In 'T Jatstraat 5, Postbus 559 (Netherlands), Netherlands

RIVERS, PROF. WILGA M., Department of Romance Lang., Harvard University, 207 Boylston Hall, Cambridge, MA 02138

ROBINSON, BEECHAM, Director, Learning Center, Wisconsin University, Kenosha, WI 53140 
ROBINSON, RICKEY, Supervisor, Media Distr. Sys., Governors State University, S. Pk Forest, IL 60466

ROEDERER, JEAN LOUIS, Professor, Houghton College, Division of Foreign Langs., Houghton, NY 14744

ROGERS, J. J., DR., Director of Modern Languages, Lincoln University, Lincoln, PA 19352

ROLFE, O. W., DR., Director of Language Lab, University of Montana, Missoula, MT 59801

ROWE, HAROLD M., Language Centre, Monash University, Clayton, Victoria 3168, Australia

ROWE, PROFESSOR GALEN O., Dept. of Foreign Langs \& Lit., College of Letters \& Science, University of Idaho, Moscow, ID 83843

ROYALTY, JAMES E., Language Media Center, University of Maryland, College Park, MD 20742

RUSDEN, SCV, Librarian, 662 North Blackburn Road, Clayton 3168, Vic., Australia

RUTGERS UNIVERSITY, Kilmer Area Library, New Brunswick, NJ 08903

SAM HOUSTON STATE UNIVERSITY, Foreign Language Department, Huntsville, TX 77340

SAMPON, VICTOR, Director Language Lab, Wayland Academy, Beaver Dam, WI 53916

SANANVANIT, MS. KWANCHI, Sea Enterprises Co., Ltd., 292/15-16 Luk Luang Road, Paris Theatre, Bangkok 3, Thailand

SANCHEZ, MARY, Supervisor Foreign Lang., Clearwater Public Schools, 1960 E. Druid Road, P.O. Box 4688, Clearwater, FL 33518

SAYLOR, LESLIE G., Language Lab, Curtin B-31, 3225 Downer Ave., Milwaukee, WI 53201

SCARTH, MARGARET, Asst. Professor-Spanish, Erindale College, University of Toronto, 3359 Mississauga Road, Clarkson, Toronto, On, Canada

SCHABACHER, RUTH, Summit High School, 129 Kent Place Blvd., Summit, NJ 07901

SCHEWE JR., EDWARD, 419 South Taylor Avcnuc. Oak Park, IL 60302

SCHOBEL, DIANNE, 7801 Sagamore Drive, Cincinnati, OH 45236

SEAMEO REG. ENG. LANG., Librarian of Info Center, 30 Orange Grove Road, Singapore, 10, Singapore

SECONDARY TEACHERS COLLEGE, Librarian, Private Bag, Auckland, 3, New Zealand

SEIDEL, J., Language Laboratory, University of Victoria, P.O. Box 1700, Victoria, BC, Canada

SENG, MARK W., Assoc. Professor, University of Texas, 2505 Princeton Drive, Austin, TX 78741

SEYDOUX, ALINE, Associate Director, Learning Resource Center, Loyola University, 7101 W. 80th Street, Los Angeles, CA 90045

SHEEHAN, JOSEPH H., School of Linguistics, Georgetown University, Washington, DC 20052

SHIRLEY, CARL R., Director F. L. Resources Center, University of South Carolina, Columbia, SC 29208 
SIMON FRASER UNIVERSITY, Library-Acquisition Div., Burnaby, BC 2, Canada

SMITH, PHILIP D., DR., Professor, Languages, West Chester State College, M 115, West Chester, PA 19380

SMITH, SHANNON W., 46 Mountfort St., Apt. 2, Boston, MA 02116

SMITH, WILLIAM FLINT, Department of Mod. Lang., Purdue University, Lafayette, IN 47907

SMITHER, WILLIAM J., Director of Language Lab, Tulane University, New Orleans, LA 70118

SNOUSE, PAT, Instructional Assistant, Davidson College, Davidson, NC 28036

SOLDEVILLE, DR. F., Director, Scripps College, Language Lab, Balch Hall No. 41, Claremont, CA 91711

SOMMER, URSULA, Asst. Dir. Language Lab, Fairleight Dickinson Univ., FlorhamMadison Campus, 285 Madison Avenue, Madison, NJ 07940

SOUTHERN ILLINOIS UNIVERSITY, Morris Lib, Periodicals Department, Carbondale, IL 62901

SOUTHWESTERN COLLEGE, Library, 900 Otay Lakes Road, Chula Vista, CA 92010

SOUTHWESTERN, UNIV. OF LOUISIANA, Serials Department, University Libraries, Lafayette, LA 70504

SPINKS, O. R., Director, Humanities B 16, Suny at Albany, Albany, NY 12203

ST. ANDREWS, UNIVERSITY of, Modern Lang. Library, Attn: Ms. Konn, Buchanan Building, Union Street, St. Andrews, Fife KY16 9PH, Canada

ST. JOHN'S UNIVERSITY, Language Laboratory, Attn: Dr. Sallese, St. Albert's Hall Rm. 103, Grand Central \& Utopia, Jamaica, NY 11439

ST. MICHAEL'S COLLEGE, Director-International Stud., Winooski, VT 05404

STADT-UND UNIVERSITATS-134-138, D-6 Frankfurt/Main-1, West Germany

STANFORD UNIVERSITY, Library-Serials Department, Stanford, CA 94305

STEELE, KATHERINE L., DR., University of Florida, Office of Instructional Res, 450 Library East, Gainesville, FL 32611

STERN, RHODA, Supervisor Language Lab, Baruch College, City University of New York, 17 Lexington Avenue, PO Box 447, New York, NY 10010

SUMMIT JR. HIGH SCHOOL, Attn: Ms. Helen Landry, 272 Morris Avenue, Summit, NJ 07901

SUTTON, LOIS M., DR., Professor of French, Baylor, University, Waco, TX 76703

SUZUKI, JUICHI, 610-205 Vematsu-Cho, Kishiwada-Shi Osaka, 596, Japan

SWANSON, SEVERIN A., Language Lab Director, University of Wisconsin, Oshkosh, WI 54091

SWIFT, MS. M. H., Morgan Park Academy, 2153 West 111th Street, Chicago, IL 60643

SWINYARD, SHARON J., Professor/Director, St. Patrick's College, Language Lab, Box 151, Mountain View, CA 94042

SYDNEY, University of Fischer Library, Serials Division 134881, Sydney, New South Wales, 2006, Australia 
TANDBERG OF AMERICA INC., President, Kjell S. Hoel, Labriola Court, Armonk, NY 10504

TAYLOR JR., WALTER C., Department of Spanish, Upsala College, East Orange, NJ 07019 TEACHERS COLLEGE, Library, 525 West 120th Street, New York, NY 10027

TENNESSEE, UNIVERSITY OF, Library Serials Division, Knoxville, TN 37916

TEXAS STATE UNIVERSITY, North Acquisitions Services, Ms. L. Evans, Box 5138 NT Station, Denton, TX 76203

TEXAS TECH UNIVERSITY, Germanic \& Slavic Languages, University Library, P.O. Box 4579, Lubbock, TX 79409

TEXAS-AUSTIN, UNIVERSITY OF, John C. Bordie, Director, Foreign Languages Education, Center Library, Austin, TX $\mathbf{7 8 7 1 2}$

TEXAS-AUSTIN, UNIVERSITY OF, Language Laboratory, Batts Hall 1, Austin, TX 78712

THERESE, MARIE SR., Director of Language Lab., College of White Plains, 78 North Broadway, White Plains, NY 10603

THIBAULT, CLAUDE, Notre Dame De Foy, Cap-Rouge, PQ GOA-1KO, Canada

THORNDIKE, KAY, Assistant Instructor, University of Nebraska, P.O. Box 688, Omaha, NE 68761

THORNE, E. F., Associate Professor, West Liberty State College, West Liberty, WV 26074

THORSTENSON, ALLEN C., Johnson Associates, 1711 W. Terra Cotta Place, Chicago, IL 60614

TOKYO-SEITOKU-TANKI-DAIGAKU, Library (MZ), 7-13, Jujodai-1-Chome, Kita-Ku, Tokyo, Japan

TOLEDO, UNIVERSITY OF, Library, 2801 W. Baneroft St., Toledo, OH 43606

TRACY, ROBERT J., Director Language Lab, Ridgewood High School, Ridgewood, NJ 07450

TUBINGEN, UNIVERSITAT KASSE, Zentrum-Neue Lernverfahren, Federal Republic of Germany, Germany

UMMEL, CAROLYN M., Valley View High School, R.R. 2 Box 3370, Germantown, OH 45327

UNION COLLEGE, Library, 3800 S. 48th Street, Lincoln, NE 68506

UNIVERSITEITSBIBLIOTHEEK, 1429, A. 324. 639, Erasmuslaan, 36, Nijmegen 1, Holland

USAF ACADEMY, Academy Library, MK For F05611-73-M-A646, CO 80840

VALACHOVIC, JARDOSLAV, 162 Westwood Road, Columbus, OH 43214

VAN MERKENSTEIJIN, E. C., Director Language Lab, University of Pennsylvania, 320 Logan Hall, Philadelphia, PA 19104

VAN WALK, GRACE R., Instructor, Grants Pass High School, 522 NE Olive, Grants Pass, OR 97526

VAN ZAANEN, RICHARD B., Director, Audio-Visual Center, Rutgers University, 392 High Street, Bradley Hall 406, Newark, NJ 07752

VEITZ, SISTER M. FRANCES, Assistant Professor, Holy Family College, Grant \& Frankfort Avenue, Philadelphia, PA 19114

VeltMAN, PROF. HUCH E., Grand Valley State College, P.O. Box 58, Lamont, MI 49430 
VERNICK, JUDY A., Director Language Lab, University of Pittsburgh, $732 \mathrm{C}$ of $\mathrm{L}$, Pittsburgh, PA 15260

VERRETTE, VICTOR S., Language Lab Director, Grinnell College, Grinnell, IA 50112

VEVERIS, GUNARS, 20 Clark Avenue, Wyoming, OH 45215

VIJAYAKUMAR, E. K., Thurber Comprehensive H. S., 15 Orilla Park, Red Deer, AB, Canada

VIKAR, A., MR., 310-629 B. Huron Street, London, ON N5Y 4J7, Canada

VIRGINIA, UNIVERSITY OF, Language Laboratory, 221 Cabell Hall, Charlottesxille, VA 22903

VITI, ROBERT M., Language Laboratory Director, Gettysburg College, German Department, Gettysburg, PA 17325

WARKENTIN, HENRY, DR., Foreign Language Department, Shippensburg State College, Shippensburg, PA 17257

WASEDA UNIVERSITY, Shoichi, Kuriyama, Japan

WASHINCTON UNIVERSITY, Library, Acquisitions Department, St. Louis, MO 63130

WATERLOO, UNIVERSITY OF, C. W. HENNING, Learning Center, Md BIdg. Room 110, Waterloo, ON, Canada

WATSON, JOHN A., DR., Director Language Lab, Virginia Union University, Richmond, VA 23220

WEATHERFORD, MS. HARRIETT, Language Lab Director, University of Illinois, G-70 Foreign Languages, Urbana, IL 61801

WEIBLE, DR. DAVID M., Director, Audio Info Service, University of Illinois at Chicago Circle, Box 4348, Chicago, IL 60680

WEINKAUf, ARNOLD L., DR., Assoc. Prof. of Languages, Michigan Technological Univ., Houghton, MI 49931

WELLESLEY, SR. HIGH SCHOOL, Foreign Language Department, 50 Rice Street, Wellesley HIs, MA 02181

WEST CHESTER STATE COLLEGE, Francis Green Library, West Chester State College, West Chester, PA 19380

WEST GEORGIA COLLEGE, School of Arts \& Science, Department of Foreign Lang., Carrollton, GA 30117

WEST TEXAS STATE UNIVERSITY, Cornette Library, Box 748 W. T. Station, Canyon, TX 79016

WESTERN ONTARIO, UNIVERSITY OF, General Library, Periodicals Department, London, ON N6A 3K7, Canada

WIESE, PETER, Director Language Lab, Southern Connecticut College, 501 Crescent Street, New Haven, CT 06515

WILKINS, RUDY III, Director Language Center, Els Language Center, 5009 Calhoun Road, Houston, TX 77004

WILLECKE, F. H., DR., Chairman Language Dept., Wagner College, Staten Island, NY 10301

WILLIAMS, CHARLES B., Director Language Lab, State University of New York, Oswego, NY 13126 
WILLIAMS, JAMES J., Director Language Lab, Elmhurst College, 190 Prospect Avenue, Elmhurst, IL 60126

WILLIS, IONE, R. R. 3, Milton, KY 40045

WILLIS, ROBERT M., Professor, Communications Arts \& Sciences, 829 Shimkin Hall, 50 W. 4th St., New York, NY 10003

WILLISTON, MRS. JACQUELINE, Director Language Lab, Connecticut College, Box 1524 New London, CT 06320

WILTON SENIOR HIGH SCHOOL, Library, 395 Danbury Road, Wilton, CT 06897

WIPF, JOSEPH, 121 East Knox Drive, W. Lafayette, IN 47906

WISCONSIN, UNIVERSITY OF, Parkside, Library, Serials Division, Wood Road, Kenosha, WI 53140

WISCONSIN, UNIVERSITY OF, Library, Serials Department, Milwaukee, WI 53201

WISCONSIN, UNIVERSITY OF, Serials Department-Library, 728 State Street, Madison, WI 53706

WOLLSTEIN, JOHN, MR., Program Specialist-For Langs, Department of Education, 1270 Queen Emma Street, Room 1201, Honolulu, HI 96813

WCOSTER, COLLEGE OF TECHNICAL SERVICES DIVISION, Andrews Library, Wooster, $\mathrm{OH} 44691$

WYNN, WALTER W., 975 Ventura Avenue, Albany, CA 94707

YANNEY, DOUGLAS M., Director Language Lab, Wichita State University, Box 11, Wichita, KS 67208

YELLIN TEACHER'S SEMINARY, Beth-Hakerem, Jerusalem, Israel

VIN, MS. PHILLIPPA B., Director Language Lab, Cleveland State University, Cleveland, $\mathrm{OH} 44115$

VLICP, TURUN, Alex Von Humbol, Kielilaboratorio, 20500 Turku 50, Findland

YORK UNIVERSITY, Scott Library, Central Serials Records, 4700 Keele Street, Downsview, ON M3) 1P3, Canada

ZARR, VALOIS A., Chairman German Department, East High School, 1134 South 17th Street East, Salt Lake City, UT 84108

ZAVATTERO, FRED H., Professor, Hosei University, Building 62, Fujimi-Cho, ChiyodaKu, Tokyo, Japan

ZENICK, MANUEL C., U.S. Peace Corps, American Embassy, P.O. Box No. PC-15-761, APO, San Francisco, CA 96346

ZIMMERMAN, DOROTHY M., SISTER, Mount Mary College, Language Lab, Milwaukee, WI 53222

ZIMMERMAN, M. MRS., Language Lab, Amherst College, Amherst, MA 01002

ZINGIS, ANDRIS, Director Language Lab, University of Cincinnati, 372 Old Chemistry Bldg., Cincinnati, $\mathrm{OH} 45221$

ZIRKO, STEPHAN, Director Language Lab, Eastern Montana College, Billings, MT 59101 